ANNALES

POLONICI MATHEMATICI

LXXVI.3 (2001)

\title{
A note on Picard iterates of nonexpansive mappings
}

\author{
by Eun Suk Kim (Pusan) and W. A. Kirk (Iowa City, IA)
}

\begin{abstract}
Let $X$ be a Banach space, $C$ a closed subset of $X$, and $T: C \rightarrow C$ a nonexpansive mapping. It has recently been shown that if $X$ is reflexive and locally uniformly convex and if the fixed point set $F(T)$ of $T$ has nonempty interior then the Picard iterates of the mapping $T$ always converge to a point of $F(T)$. In this paper it is shown that if $T$ is assumed to be asymptotically regular, this condition can be weakened much further. Finally, some observations are made about the geometric conditions imposed.
\end{abstract}

1. Introduction. The purpose of this paper is to further investigate convergence of Picard iterates of nonexpansive mappings whose fixed point sets have nonempty interior. In $1978 \mathrm{~J}$. Moreau [11] obtained the first result of this type by proving that if $C$ is a closed subset of a Hilbert space $H$, if $T$ : $C \rightarrow C$ is nonexpansive, and if the fixed point set $F(T)$ of $T$ has nonempty interior, then for every $x \in C$ the Picard iterates of $T$ at $x$ converge strongly to a point of $F(T)$. Subsequently, B. Beauzamy observed that this result also holds in uniformly convex spaces; see e.g. [5], pp. 219-222, for a proof. In [9] it is shown that this fact extends even to reflexive locally uniformly convex spaces. It is also shown in [9] that if $T$ is asymptotically regular, then the Picard iterates of $T$ converge to a fixed point of $T$ under the assumption that the underlying space has Rolewicz's property $(\beta)$. It follows that if $C$ is a convex subset of such a space and if $f$ is an "averaged mapping" of $T: C \rightarrow C$ (thus $f=(1-t) I+t T$ for some $t \in(0,1))$, then the Picard iterates of $f$ converge to a fixed point of $T$ whenever $\operatorname{int}(F(T)) \neq \emptyset$. Our main objective is to extend this latter fact.

We begin with some definitions and basic facts. Throughout, $X$ will denote a Banach space. We use $B_{X}$ and $S_{X}$ to denote the closed unit ball and unit sphere of $X$ respectively. For brevity, $B_{r}$ will denote the closed ball centered at the origin with radius $r \geq 0$, and $S_{r}$ the surface of $B_{r}$. Thus

2000 Mathematics Subject Classification: Primary 47H10, 46B20.

Key words and phrases: fixed points, nonexpansive mappings, Picard iterates.

This research was carried out while the first author was visiting the University of Iowa with the support of a KOSEF grant. 
$S_{X}=\{x \in X:\|x\|=1\}$ and, for $r \neq 1, S_{r}=\{x \in X:\|x\|=r\}$. In general, we use $B(x ; r)$ and $S(x ; r)$ to denote respectively the closed ball and sphere centered at $x \in X$ of radius $r$.

For later reference we need the following definition. lar if

Definition 1. A mapping $T: C \rightarrow C$ is said to be asymptotically regu-

$$
\lim _{n \rightarrow \infty}\left\|T^{n}(x)-T^{n+1}(x)\right\|=0 \quad \text { for all } x \in C .
$$

A mapping $T: C \rightarrow C$ is said to be nonexpansive if $\|T(x)-T(y)\| \leq$ $\|x-y\|$ for every $x, y \in C$. Asymptotic regularity arises naturally in the study of nonexpansive mappings. If $T: C \rightarrow C$ is nonexpansive and if $C$ is bounded and convex, then a fundamental result of Ishikawa [4] assures that for any $t \in(0,1)$ the mapping $T_{t}: C \rightarrow C$ defined by

$$
T_{t}(x)=(1-t) x+t T(x), \quad x \in C,
$$

is always asymptotically regular. Moreover, $F\left(T_{t}\right)=F(T)$.

Our results concerning asymptotically regular mappings will depend on two well known elementary facts. Suppose $C$ is a closed subset of $X$ and $T: C \rightarrow C$.

REMARK 1. If $T$ is nonexpansive and $\lim _{k \rightarrow \infty} T^{n_{k}}(x)=z \in F(T)$, then $\lim _{n \rightarrow \infty} T^{n}(x)=z$.

REMARK 2. If $T$ is continuous and asymptotically regular, and if $\lim _{k \rightarrow \infty} T^{n_{k}}(x)=z$, then $z \in F(T)$.

2. Preliminaries. The definitions introduced below will be discussed in slightly more detail later. These are in some sense motivated by the following two propositions. The first proposition seems to be due independently to Edelstein [1] and Stechkin [13].

Proposition 1. A Banach space $X$ is uniformly convex if and only if for fixed positive numbers $\varepsilon, \varrho$, and $r<\varrho$ there exists $\xi=\xi(\varepsilon, \varrho, r)>0$ such that if $u \in X$ satisfies $\|u\|=r$ then

$$
\operatorname{diam}(B(u ; \varrho-r+\xi) \cap(X \backslash B(0 ; \varrho))<\varepsilon .
$$

Our second proposition involves Rolewicz's property $(\beta)$. Recall that for $x \in X \backslash B_{X}$, the drop of $x$ is the set

$$
R\left(x, B_{X}\right)=D\left(x, B_{X}\right) \backslash B_{X}
$$

where $D\left(x, B_{X}\right)=\operatorname{conv}\left(\{x\} \cup B_{X}\right)$. In the following definition, $\alpha$ denotes the usual Kuratowski measure of noncompactness. 
Definition 2. A Banach space $X$ is said to have property $(\beta)$ if for each $\varepsilon>0$ there exists $\delta>0$ such that if $x \in X \backslash B_{X}$ satisfies $1<\|x\|<1+\delta$, then $\alpha\left[R\left(x, B_{X}\right)\right]<\varepsilon$.

The following fact is proved in [6].

Proposition 2. A Banach space $X$ has property $(\beta)$ if and only if given positive numbers $\varepsilon, \varrho$, and $r<\varrho$ there exists $\xi=\xi(\varepsilon, \varrho, r)>0$ such that if $u \in X$ satisfies $\|u\|=r$ then

$$
\alpha[B(u ; \varrho-r+\xi) \cap(X \backslash B(0 ; \varrho)] \leq \varepsilon .
$$

For our extensions we make use of the notion of " $k$-dimensional volume" as discussed in [3] and [14]. For $\left\{x_{1}, \ldots, x_{k+1}\right\}$ in a Banach space $X$, set

$$
V\left(x_{1}, \ldots, x_{k+1}\right)=\sup \left\{\left|\begin{array}{ccc}
1 & \ldots & 1 \\
f_{1}\left(x_{1}\right) & \ldots & f_{1}\left(x_{k+1}\right) \\
\vdots & & \vdots \\
f_{k}\left(x_{1}\right) & \ldots & f_{k}\left(x_{k+1}\right)
\end{array}\right|: f_{i} \in S_{X^{*}}, i=1, \ldots, k\right\} \text {. }
$$

A proof of the following proposition can be found in Kirk [8].

Proposition 3. Let $\left\{x_{n}\right\}$ be a bounded sequence in $X$ with $\left\|x_{n}-x_{m}\right\|$ $\geq \varepsilon>0$ for $n \neq m$. Then given any $k \in \mathbb{N}$ and any $\lambda \in(0, \varepsilon)$ there exists a subsequence $\left\{z_{n}\right\}$ of $\left\{x_{n}\right\}$ such that if $\left\{z_{n_{1}}, \ldots, z_{n_{k+1}}\right\}$ is any set of $k+1$ distinct points of $\left\{z_{n}\right\}$, then

$$
V\left(z_{n_{1}}, \ldots, z_{n_{k+1}}\right) \geq(\lambda / 2)^{k-1} .
$$

The following condition is clearly weaker that the assumption of uniform convexity (for $k>0$ ).

Definition 3. A Banach space $X$ is said to have the $k$-spherical cap $(k-(S C))$ property, $k \geq 0$, if the following is true. For each $\varepsilon>0, \varrho>0$, and $r \in(0, \varrho)$ there exist $\xi=\xi(\varepsilon, \varrho, r)>0$ such that if $\left\{x_{1}, \ldots, x_{k+1}\right\} \subset B_{X}$ satisfies $\left\|x_{i}\right\|=r$, and $V\left(x_{1}, \ldots, x_{k+1}\right) \geq \varepsilon$, then

$$
\operatorname{diam}\left[\left(\bigcap_{i=1}^{k+1} B\left(x_{i} ; \varrho-r+\xi\right)\right) \cap(X \backslash B(0 ; \varrho))\right] \leq \varepsilon .
$$

Our extension of the concept of property $(\beta)$ is the following.

Definition 4. A Banach space $X$ is said to have property $k-(\beta), k \in \mathbb{N}$, if the following is true. For each $\varepsilon>0, \varrho>0$, and $r \in(0, \varrho)$ there exist $\xi=\xi(\varepsilon, \varrho, r)>0$ such that if $\left\{x_{1}, \ldots, x_{k+1}\right\} \subset B_{X}$ satisfies $\left\|x_{i}\right\|=r$, and $V\left(x_{1}, \ldots, x_{k+1}\right) \geq \varepsilon$, then

$$
\alpha\left[\left(\bigcap_{i=1}^{k} B\left(x_{i} ; \varrho-r+\xi\right)\right) \cap(X \backslash B(0 ; \varrho))\right] \leq \varepsilon .
$$


3. Main results. Our main observation is the following.

Theorem 1. Suppose $C$ is a closed convex subset of a Banach space which has property $k-(\beta)$ for some $k \in \mathbb{N}$, and suppose $T: C \rightarrow C$ is a nonexpansive mapping which is also asymptotically regular. Suppose moreover that the fixed point set of $T$ has nonempty interior. Then the Picard iterates $\left\{T^{n}(x)\right\}$ converge to a fixed point of $T$ for each $x \in C$.

Proof. Select $p \in \operatorname{int}(F(T))$, choose $r>0$ so that $B(p ; r) \subset F(T)$, and let $x \in C$. Since $T$ is nonexpansive we have $\lim _{n \rightarrow \infty}\left\|p-T^{n}(x)\right\|=\varrho \geq r$, and if $\varrho=r$ there is nothing to prove. So we assume $\varrho>r$, and for each $n \in \mathbb{N}$ choose $u_{n} \in \operatorname{Fix}(T)$ so that $\left\|p-u_{n}\right\|=r, u_{n} \in \operatorname{seg}\left[p, T^{n}(x)\right]$, and

$$
\left\|p-u_{n}\right\|+\left\|u_{n}-T^{n}(x)\right\|=\left\|p-T^{n}(x)\right\| .
$$

In view of Remarks 1 and 2 it need only be shown that some subsequence of $\left\{T^{n}(x)\right\}$ converges. If not, then there exists $\varepsilon>0$ such that

$$
\alpha\left[x, T(x), T^{2}(x), \ldots\right] \geq \varepsilon
$$

and by Proposition 3 it is possible to choose $0<\varepsilon^{\prime}<\varepsilon$ and a subsequence $\left\{u_{n_{i}}\right\}$ of $\left\{u_{n}\right\}$ such that

$$
V\left(u_{n_{1}}, \ldots, u_{n_{k+1}}\right) \geq \varepsilon^{\prime} .
$$

Now let $\xi=\xi\left(\varepsilon^{\prime}, \varrho, r\right)$ and observe that there exists $N_{\xi} \in \mathbb{N}$ such that if $n \geq N_{\xi}$ then

$$
\left\|p-T^{n}(x)\right\| \leq \varrho+\xi
$$

Therefore if $m>n \geq N_{\xi}$ then

$$
\begin{aligned}
\left\|u_{n}-T^{m}(x)\right\| & =\left\|T^{m-n}\left(u_{n}\right)-T^{m-n} \circ T^{n}(x)\right\| \leq\left\|u_{n}-T^{n}(x)\right\| \\
& =\left\|p-T^{n}(x)\right\|-\left\|p-u_{n}\right\| \leq \varrho+\xi-r .
\end{aligned}
$$

In particular, $n \geq N_{\xi}$ and if $i \geq m$, then

$$
T^{i}(x) \in B\left(u_{n} ; \varrho+\xi-r\right) \cap(X \backslash B(p ; \varrho)) .
$$

Now choose $k^{\prime} \geq k+1$ so large that at least $k+1$ distinct terms of the sequence $\left\{u_{n_{i}}\right\}$ lie in the set $\left\{u_{N_{\xi}}, \ldots, u_{N_{\xi}+k^{\prime}}\right\}$. Then if $m \geq N_{\xi}+k^{\prime}$ we have

$$
T^{m}(x) \in\left(\bigcap_{i=N_{\xi}}^{N_{\xi}+k^{\prime}} B\left(u_{i} ; \varrho+\xi-r\right)\right) \cap(X \backslash B(p ; \varrho)) .
$$

But

$$
\alpha\left[\left(\bigcap_{i=N_{\xi}}^{N_{\xi}+k^{\prime}} B\left(u_{i} ; \varrho+\xi-r\right)\right) \cap(X \backslash B(p ; \varrho))\right] \leq \varepsilon^{\prime}<\varepsilon
$$

and this contradicts

$$
\alpha\left[T^{m}(x), T^{m+1}(x), \ldots\right]=\alpha\left[x, T(x), T^{2}(x), \ldots\right] \geq \varepsilon .
$$


Corollary 1. Suppose $C$ is a closed convex subset of a Banach space which has property $k-(\beta)$ for some $k \in \mathbb{N}$, and suppose $T: C \rightarrow C$ is a nonexpansive mapping. Suppose also that the fixed point set of $T$ has nonempty interior. Set $T_{\lambda}:=(1-\lambda) I+\lambda T, \lambda \in(0,1)$. Then the Picard iterates $\left\{T_{\lambda}^{n}(x)\right\}$ converge to a fixed point of $T$ for each $x \in C$.

Proof. By a well known result of Ishikawa [4], $T_{\lambda}$ is asymptotically regular.

A slight modification of the above approach yields the following result for Picard iterates of mappings $T$ which need not be assumed asymptotically regular. At this point the significance of the geometric hypothesis is not clear but at least formally it appears to be weaker than uniform convexity.

Theorem 2. Suppose $C$ is a closed subset of a Banach space which has the 1-spherical cap property, and suppose $T: C \rightarrow C$ is a nonexpansive mapping. Suppose also that the fixed point set of $T$ has nonempty interior. Then the Picard iterates $\left\{T^{n}(x)\right\}$ converge to a fixed point of $T$ for each $x \in C$.

Proof. Select $p \in \operatorname{int}(\operatorname{Fix}(T))$, choose $r>0$ so that $B(p ; r) \subset \operatorname{Fix}(T)$, and let $x \in C$. Let $\lim _{n \rightarrow \infty}\left\|p-T^{n}(x)\right\|=\varrho \geq r$, and for each $n \in \mathbb{N}$ choose $u_{n} \in \operatorname{Fix}(T)$ as in the proof of Theorem 1 .

Now for each $\xi>0$ there exists $N_{\xi} \in \mathbb{N}$ so that if $n \geq N_{\xi}$ then

$$
\left\|p-T^{n}(x)\right\| \leq \varrho+\xi .
$$

Then if $m>n \geq N_{\xi}$ we have

$$
\begin{aligned}
\left\|u_{n}-T^{m}(x)\right\| & =\left\|T^{m-n}\left(u_{n}\right)-T^{m-n} \circ T^{n}(x)\right\| \leq\left\|u_{n}-T^{n}(x)\right\| \\
& =\left\|p-T^{n}(x)\right\|-\left\|p-u_{n}\right\| \leq \varrho+\xi-r .
\end{aligned}
$$

In particular, $m>n \geq N_{\xi}$ and if $i \geq m$, then

$$
T^{i}(x) \in\left[B\left(u_{n} ; \varrho+\xi-r\right) \cap B\left(u_{m} ; \varrho+\xi-r\right)\right] \cap(X \backslash B(p ; \varrho)) .
$$

Now assume $\left\{T^{n}(x)\right\}$ is not Cauchy. Then there exists $\varepsilon>0$ such that for any $n \in \mathbb{N}$ there exist $i, j \geq n$ for which

$$
\left\|T^{i}(x)-T^{j}(x)\right\| \geq \varepsilon .
$$

For each such pair $i, j$ there exists a fixed $\varepsilon^{\prime}>0$, independent of $i$ and $j$ and with $\varepsilon^{\prime}<\varepsilon$, such that $\left\|u_{i}-u_{j}\right\| \geq \varepsilon^{\prime}$. Set $\xi=\xi\left(\varepsilon^{\prime}, \varrho, r\right)$ and choose $i, j \geq m>n \geq N_{\xi}$ so that $\left\|T^{i}(x)-T^{j}(x)\right\| \geq \varepsilon$. Since $X$ has the 1 -spherical cap property,

$$
\left.\operatorname{diam}\left(\left[B\left(u_{n} ; \varrho+\xi-r\right)\right) \cap B\left(u_{m} ; \varrho+\xi-r\right)\right] \cap(X \backslash B(p ; \varrho))\right) \leq \varepsilon^{\prime}<\varepsilon
$$

and this is a contradiction.

4. An extension. The results of [9] hinge on the fact that the closed subsets of the underlying space are densely proximinal. This means that for 
any nonempty closed subset $F$ of $X$ the set

$$
\left\{x \in X: \exists p_{x} \in F \text { such that }\left\|x-p_{x}\right\|=\operatorname{dist}(x, F)\right\}
$$

is dense in $X$. For this reason it is not known whether the approach of [9] applies in the settings of this paper. In particular, it is known that closed subsets of spaces with property $(\beta)$ are densely proximinal. This raises the obvious question of whether nonempty closed subsets of spaces with property $k-(\beta)$ are also densely proximinal.

We now give a minor extension of one of the principal results of KirkSims [9]. It is shown in [9] (Theorem 2.2) that if $X$ is a Banach space whose norm is strictly convex and whose closed subsets are densely proximinal and if $T$ is a nonexpansive mapping of a closed subset $C$ of $X$ into itself for which $\operatorname{int}(F(T)) \neq \emptyset$, then the Picard sequence $\left\{T^{n}(x)\right\}$ converges to a fixed point of $T$ for every $x \in C$. As we see below this result extends easily to mappings $T$ which are eventually nonexpansive, that is, mappings $T$ for which $T^{n}$ is nonexpansive for all $n \geq N$ for some fixed $N \in \mathbb{N}$. In particular, such mappings need not even be continuous. It is noted in [7] that for a large class of spaces the fixed point property for nonexpansive mappings coincides with the fixed point property for eventually nonexpansive mappings.

TheOREM 3. Let $X$ be a Banach space whose norm is strictly convex and whose nonempty closed subsets are densely proximinal. (Alternatively, suppose $X$ satisfies the assumptions of Theorem 2.) Let $C$ be a closed subset of $X$ and let $T: C \rightarrow C$ be eventually nonexpansive with $\operatorname{int}(F(T)) \neq \emptyset$. Then for each $x \in C$ the Picard sequence $\left\{T^{n}(x)\right\}$ converges to a fixed point of $T$.

Proof. Since $T$ is eventually nonexpansive there exists $N \in \mathbb{N}$ such that both $T^{N}$ and $T^{N+1}$ are nonexpansive. Also, since $F(T) \subseteq F\left(T^{N}\right)$ and $F(T) \subseteq F\left(T^{N+1}\right)$, the fixed point sets of $T^{N}$ and $T^{N+1}$ both have nonempty interior. Let $x \in C$. By Theorem 2.2 of [9] (alternatively, by Theorem 2) there exist $z_{1} \in F\left(T^{N}\right)$ and $z_{2} \in F\left(T^{N+1}\right)$ such that

$$
\lim _{k \rightarrow \infty} T^{k N}(x)=z_{1} \quad \text { and } \quad \lim _{k \rightarrow \infty} T^{k(N+1)}(x)=z_{2} .
$$

Since $\left\{T^{k N(N+1)}(x)\right\}_{k=1}^{\infty}$ is a common subsequence of both $\left\{T^{k N}(x)\right\}_{k=1}^{\infty}$ and $\left\{T^{k(N+1)}(x)\right\}_{k=1}^{\infty}$ it follows that $z_{1}=z_{2}$. Hence $z:=z_{1}=z_{2} \in F\left(T^{N}\right) \cap$ $F\left(T^{N+1}\right)=F(T)$ (Lemma 1 of [7]). Let $\varepsilon>0$ and choose $k \in \mathbb{N}$ so that

$$
\left\|T^{k N}(x)-z\right\| \leq \varepsilon .
$$

Then if $n \geq(k+1) N$, say $n=(k+1) N+q$, we have

$$
\left\|T^{n}(x)-z\right\|=\left\|T^{(k+1) N+q}(x)-T^{N+q}(z)\right\| \leq\left\|T^{k N}(x)-z\right\| \leq \varepsilon .
$$

Thus $\lim _{n \rightarrow \infty} T^{n}(x)=z$.

COROllary 2. Let $X$ be a reflexive locally uniformly convex Banach space and let $C$ be a closed subset of $X$. Suppose $T: C \rightarrow C$ is eventually 
nonexpansive with $\operatorname{int}(F(T)) \neq \emptyset$. Then for each $x \in C$ the Picard sequence $\left\{T^{n}(x)\right\}$ converges to a point of $F(T)$.

Proof. Nonempty closed subsets of reflexive locally uniformly convex Banach spaces are densely proximinal (Lau [10], Theorem 5). Since such spaces have strictly convex norm the result follows from Theorem 3.

5. An example. The assumption that $\operatorname{int}(F(T)) \neq \emptyset$ in the preceding results is of course quite strong. However, even in the nicest of spaces, say $\ell_{2}$, a simple rotation shows that some such assumption is necessary for the validity of Theorem 3, and a more complicated example by Genel and Lindenstrauss [2] shows that this is true even if the mapping is also assumed to be asymptotically regular.

At the same time the assumption $\operatorname{int}(F(T)) \neq \emptyset$ alone is in general not sufficient. Let $B$ denote the unit ball in $\ell_{\infty}$ and consider the mapping $T: B \rightarrow B$ defined as follows. For each $n$ define $\phi_{n}:[-1,1] \rightarrow[-1,1]$ by setting

$$
\phi_{n}(t)= \begin{cases}t & \text { if }|t| \leq 1 / 2, \\ \frac{1}{2 n}+\left(1-\frac{1}{n}\right) t & \text { if } 1 / 2<t \leq 1, \\ -\left(\frac{1}{2 n}+\left(1-\frac{1}{n}\right) t\right) & \text { if }-1 \leq t<-1 / 2 .\end{cases}
$$

Now for each $x:=\left(x_{1}, x_{2}, \ldots, x_{n}, \ldots\right) \in B$ define

$$
T(x)=\left(\phi_{1}\left(x_{1}\right), \phi_{2}\left(x_{2}\right), \ldots, \phi_{i}\left(x_{i}\right), \ldots\right) .
$$

It is easy to check that $\left|\phi_{n}(u)-\phi_{n}(v)\right| \leq|u-v|$ for each $n \geq 1$ and $u, v \in[-1,1]$. Thus $T$ is nonexpansive, and moreover the fixed point set $F(T)$ of $T$ is precisely the closed ball $B(0 ; 1 / 2)$. On the other hand, if

$$
x=(1,1,1, \ldots)
$$

then

$$
T(x)=\left(\frac{1}{2}, \frac{3}{4}, \ldots, \frac{2 i-1}{2 i}, \ldots\right) .
$$

Thus $\operatorname{dist}(T(x), F(T))=1 / 2$. Moreover, since $\lim _{i \rightarrow \infty} \phi_{i}^{n}(1)=1$ for each $n \geq 1, \operatorname{dist}\left(T^{n}(x), F(T)\right)=1 / 2$, and thus $\left\{T^{n}(x)\right\}$ cannot converge to a point of $F(T)$.

In fact, it is easy to check that the mapping $T$ of this example is also asymptotically regular.

Acknowledgements. We thank the referee for calling attention to several misprints in the original version of this manuscript. 


\section{References}

[1] M. Edelstein, On nearest points of sets in uniformly convex Banach spaces, J. London Math. Soc. 43 (1968), 375-377.

[2] A. Genel and J. Lindenstrauss, An example concerning fixed points, Israel J. Math. 22 (1975), 81-86.

[3] R. Geremia and F. Sullivan, Multi-dimensional volumes and moduli of convexity in Banach spaces, Ann. Mat. Pura Appl. 127 (1981), 231-251.

[4] S. Ishikawa, Fixed points and iteration of a nonexpansive mapping in a Banach space, Proc. Amer. Math. Soc. 59 (1976), 65-71.

[5] V. I. Istrăţescu, Fixed Point Theory, D. Reidel, Dordrecht, 1981.

[6] W. A. Kirk, Property $(\beta)$ and Edelstein's algorithms for constructing nearest and farthest points, in: Banach Spaces, B. L. Lin (ed.), Contemp. Math. 144, Amer. Math. Soc., 1993, 149-158.

[7] - The fixed point property and mappings which are eventually nonexpansive, in: Theory and Applications of Nonlinear Operators of Accretive and Monotone Type, A. G. Kartasatos (ed.), Marcel Dekker, New York, 1996, 141-147.

[8] -, The modulus of k-rotundity, Boll. Un. Mat. Ital. A (7) 2 (1988), 195-201.

[9] W. A. Kirk and B. Sims, Convergence of Picard iterates of nonexpansive mappings, Bull. Polish Acad. Sci. Math. 47 (1999), 147-155.

[10] K. S. Lau, Almost Chebyshev subsets in reflexive Banach spaces, Indiana Univ. Math. J. 27 (1978), 791-795.

[11] J. Moreau, Un cas de convergence des itérées d'une contraction d'un espace hilbertien, C. R. Acad. Sci. Paris Sér. 286 (1978), 143-144.

[12] S. Rolewicz, On $\Delta$ uniform convexity and drop property, Studia Math. 87 (1987), 181-191.

[13] S. B. Stečkin [S. B. Stechkin], Approximation properties of sets in normed linear spaces, Rev. Roumaine Math. Pures Appl. 8 (1963), 5-18 (in Russian).

[14] F. Sullivan, A generalization of uniformly rotund Banach spaces, Canad. J. Math. 31 (1979), 628-636.

Department of Applied Mathematics

Pukyong National University

Pusan 608-737, South Korea
Department of Mathematics The University of Iowa Iowa City, IA 52242-1419, U.S.A.

E-mail: kirk@math.uiowa.edu

Reçu par la Rédaction le 25.3.1999

Révisé le 10.10.2000 